\title{
Recurrence of fatal venous air embolism
}

\author{
Kyong Shil Im, Joon Suk Woo, and Jae Myeong Lee \\ Department of Anesthesiology and Pain Medicine, Uijeongbu St. Mary’s Hospital, The Catholic University of Korea College of \\ Medicine, Uijeongbu, Korea
}

Venous air embolism (VAE) often occurs in the operating theatre. Squeals have not been reported much in most recovering patients. Our patient recovered from a venous air embolism during surgery but experienced cardiac arrest in the intensive care unit the next day, and died. A review of the chest X-ray revealed pulmonary edema and intracardiac air.

A 42-year-old male patient was admitted for surgery on a right occipital brain meningioma. The patient had no specific medical history. The right subclavian vein was cannulated during the preoperative period. In the operating theatre, non-invasive blood pressure, electrocardiograph, pulse oximeter $\left(\mathrm{SpO}_{2}\right)$, end-tidal carbon dioxide $\left(\mathrm{ETCO}_{2}\right)$ and central venous pressure (CVP) monitoring were initiated. Anesthesia was maintained with oxygen, air, target-controlled infusion of remifentanil and propofol, and rocuronium with controlled ventilation. The left dorsalis pedis artery was cannulated for continuous arterial blood pressure monitoring (ABP). The surgery was performed in the semi-sitting position.

One hour after beginning the surgery, an abrupt CVP elevation from 8 to $18 \mathrm{cmH}_{2} \mathrm{O}$ was detected. $\mathrm{ETCO}_{2}$ decreased from 20 to $14 \mathrm{mmHg}$, and systolic ABP decreased from 110 to 92 $\mathrm{mmHg}$. After injecting $5 \mathrm{mg}$ ephedrine, blood pressure increased but decreased again. Although we suspected a pneumothorax during cannulation of the central line, both lung sounds were symmetrical and a post-procedural X-ray did not reveal a pneumothorax. Sinuses had not been cut yet, but under strong suspicion of a VAE, BP decreased, heart rate (HR) slowed, and ventricular premature complexes occurred. Surgeon sprayed water over the operative field and wrapped the wound with wet surgical pads. The surgery was stopped and the patient was repositioned in the head down position. Aspiration of blood through the central line showed air bubbles. The vital signs stabilized after 1,000 $\mathrm{ml}$ of mixed blood and air were aspirated. The cardiologist confirmed that the right heart was enlarged and deviated to the left side on a trans-thoracic echocardiograph (TTE). However, no intracardiac air was detected. When the surgery was being delayed and the surgeon sutured the wound, vital signs became unstable again. We aspirated air from the central line again, and vital signs stabilized. After wound closure, a trans-esophageal echocardiograph (TEE) revealed no intracardiac air. Intracardiac shunts such as foramen ovale were also not detected in TEE. The patient was moved to the ICU without extubation.

In the ICU, BP was 140/70 mmHg, HR, 88 beats/min; CVP, $3 \mathrm{cmH}_{2} \mathrm{O}$; and $\mathrm{SpO}_{2}, 100 \%$. The ventilator was connected with a tidal volume of $450 \mathrm{ml}$, respiratory rate of 16 breaths/min, fraction of inspiratory oxygen of 1.0, a positive end-expiratory pressure (PEEP) of $5 \mathrm{cmH}_{2} \mathrm{O}$. After a chest X-ray revealed pulmonary edema, the surgeon injected furosemide $20 \mathrm{mg}$. The Glasgow coma scale score recovered to 9 and after 1 and half hour the patient removed the endotracheal tube accidently. A rebreathing mask with $10 \mathrm{~L} / \mathrm{min}_{2}$ was used, and $\mathrm{SpO}_{2} 90 \%$ was adjusted to $90 \%$. After $30 \mathrm{~min}, \mathrm{SpO}_{2}$ decreased to $85 \%$ so the patient was reintubated and a diuretic infusion was started. Vital signs were; BP, 140/80 mmHg; HR, $150 \mathrm{bpm}$; PEEP increased to $7 \mathrm{cmH}_{2} \mathrm{O}$, and fluid was restricted. BP dropped to $70 / 50 \mathrm{mmHg}$ and a norepinephrine infusion was started $2 \mathrm{hr}$ later. A chest anteroposterior view and brain computed tomography (CT) scan were conducted. The brain CT revealed no other abnormality. Although we increased the norepinephrine dosage, the patient

Corresponding author: Jae Myeong Lee, M.D., Department of Anesthesiology and Pain Medicine, Uijeongbu St. Mary's Hospital, The Catholic University of Korea, 271, Cheon bo-ro, Uijeongbu 480-717, Korea. Tel: 82-31-820-3087, Fax: 82-31-821-8449, E-mail: jaemng@catholic.ac.kr () This is an open-access article distributed under the terms of the Creative Commons Attribution Non-Commercial License (http:// creativecommons.org/licenses/by-nc/3.0/), which permits unrestricted non-commercial use, distribution, and reproduction in any medium, provided the original work is properly cited. 


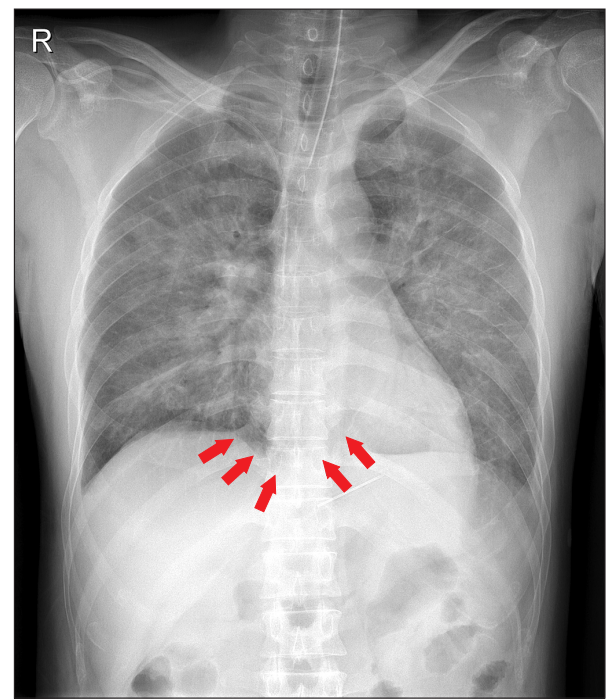

Fig. 1. A chest X-ray 7 hour after the operation reveals a huge volume of intracardiac air (arrows).

arrested and died 18 hour after surgery. We didn't suspect the air embolism in the ICU before the patient passed away. We could find the embolism in the X-ray only in the post mortem workup (Fig. 1). Follow-up echocardiogram was not done in the postoperative period. Negative intraoperative fluid input/output and within-normal range coagulation profiles were seen in the patient.

Our patient was resuscitated successfully from VAE in the operating theatre. After complete coverage of the wound by the surgeon, we managed to confirm that there was no intracardiac air in the patient by TEE. However, pulmonary edema occurred later. It was likely that fluid restriction, diuretics, and PEEP may have forced the remnant air into the cardiac chamber. The patient was dehydrated and PEEP causes pulmonary capillary to constrict and thereby increases the pulmonary vascular resistance so any air embolism can get trapped in the right heart. We should have increased intravascular volume to increase venous pressure and venous return, inotropic support and mechanical ventilation after the event [1]. There was no evidence of air entry by any other routes like central line.

Pulmonary edema after VAE is not well known. However, large volumes of air can obstruct the pulmonary vessels and diminish effective pulmonary circulation. An inflammatory reaction occurs besides the mechanical obstruction. Activation of the intrinsic coagulation pathway [2], increased microvascular permeability [3], endothelin-1 induced pulmonary hypertension [4], and release of vasoactive mediator induced systemic inflammatory response syndrome-like reaction occurs.

A chest X-ray shows air in the pulmonary artery, pulmonary edema, subcutaneous and mediastinal emphysema, focal oligemia, atelectasis, pulmonary infarction, air in the hepatic venous circulation, or intracardiac air [5]. The radiological findings of post-embolic view are important, as air bubbles can remain in the intravascular space more than several hours.

In severe cases, hyperbaric oxygen should be introduced, but in our case the patient was resuscitated successfully, and we did not believe that our patient needed hyperbaric oxygen. However, we overlooked the possibility of intravascular air remaining. We should have paid attention to the remnant air in the vessel.

Many air embolisms have been reported but specific ICU care has rarely been reported. We recommend critical care and radiological confirmations during the postoperative periods. Further exams and managements should be considered considering the remaining air. More careful concern after this type of event is required.

\section{References}

1. Muth CM, Shank ES. Gas embolism. N Eng J Med 2000; 342: 476-82.

2. Albertine KH, Wiener Kronish JP, Koike K, Staub NC. Quantification of damage by air emboli to lung microvessels in anesthetized sheep. J Appl Physiol 1984; 57: 1360-8.

3. Takeoka M, Sakai A, Ueda G, Ge RL, Panos RJ, Taniguchi S. Influence of hypoxia and pulmonary air embolism on lung injury in perfused rat lungs. Respiration 1996; 63: 346-51.

4. Tanus-Santos JE, Gordo WM, Udelsmann A, Cittadino MH, Moreno H Jr. Nonselective endothelin-receptor antagonism attenuates hemodynamic changes after massive pulmonary air embolism in dogs. Chest 2000; 118: 175-9.

5. Kizer KW, Goodman PC. Radiographic manifestations of venous air embolism. Radiology 1982; 144: 35-9. 